\title{
A universal route to pattern formation in multicellular systems
}

\author{
Malbor Asllani ${ }^{1}$, Timoteo Carletti ${ }^{2}$, Duccio Fanelli ${ }^{3}$, Philip K. Maini ${ }^{4}$ \\ ${ }^{1}$ MACSI, Department of Mathematics and Statistics, \\ University of Limerick, Limerick V94 T9PX, Ireland \\ ${ }^{2}$ Department of Mathematics and naXys, Namur Institute for Complex Systems, \\ University of Namur, rempart de la Vierge 8, B 5000 Namur, Belgium \\ ${ }^{3}$ Dipartimento di Fisica e Astronomia, Università di Firenze, \\ INFN and CSDC, Via Sansone 1, 50019 Sesto Fiorentino, Firenze, Italy and \\ ${ }^{4}$ Mathematical Institute, University of Oxford, Woodstock Rd, OX2 6GG Oxford, UK
}

\begin{abstract}
A general framework for the generation of long wavelength patterns in multi-cellular (discrete) systems is proposed, which extends beyond conventional reaction-diffusion (continuum) paradigms. The standard partial differential equations framework of reaction-diffusion type can be considered as a mean-field like ansatz which corresponds, in the biological setting, to sending to zero the size (or volume) of each individual cell. By relaxing this approximation and provided a directionality in the flux is allowed for, we here demonstrate that the instability that anticipates the ensuing patterns can always develop if the (discrete) system is large enough, namely composed by sufficiently many cells, the units of spatial patchiness. Macroscopic patterns that follow the onset of the instability are robust and show oscillatory or steady state behaviour.
\end{abstract}

\section{INTRODUCTION}

Self-organization, the ability of a system of microscopically interacting entities to shape macroscopically ordered structures, is ubiquitous in Nature. Spatiotemporal patterns are abundantly observed in a large plethora of applications, encompassing different fields and scales. Examples of emerging patterns are the spots and stripes on the coat or skin of animals $[1,2]$, the spatial distribution of vegetation in arid areas [3], the organization of the colonies of insects in host-parasitoid systems [4] and the architecture of large complex ecosystems [5]. In the early $1950^{\prime} s$, Alan Turing laid down, in a seminal paper [6], the mathematical basis of pattern formation, the discipline that aims at explaining the richness and diversity of forms displayed in Nature. Turing's idea paved the way for a whole field of investigation and fertilized a cross-disciplinary perspective to yield a universally accepted paradigm of self-organization [7]. The onset of pattern formation on a bound spatial domain originates from the loss of stability of an unpatterned equilibrium. To start with, Turing proposed a minimal model composed of at least two chemicals, hereby termed species. The species were assumed to diffuse across an ensemble of cells, adjacent to each other and organized in a closed ring, as depicted in Figure 1 a). One of the species should trigger its own growth, acting therefore as a self-catalyst. This is opposed by the competing species, which therefore promotes an effective stabilization of the underlying dynamics. The emergence of the ensuing spatial order relies on these contrasting interactions and requires, as an unavoidable constraint, a marked difference (as measured by the ratio) of the diffusion constants associated with the interacting species [8]. This symmetrybreaking mechanism is at the core of a general principle, widely known as the Turing instability [9]: small inhomogeneous perturbations from a uniform steady state initi- ate the instability and individuals, in a quest for space and resources, organise in spatially extended, regular motifs [10]. This original idea was built upon by Meinhardt [11], who proposed the notion of activators and inhibitors, so that the Turing patterning principle could be conceptualized as arising through short-range activation (slow diffusion), long-range inhibition (fast diffusion).

Pattern formation for systems evolving on cellular arrays was further analyzed by Othmer and Scriven [12] under the assumption of symmetric diffusion. Agregates of cells yield macroscopic tissues which, in general, can be schematized, to a reasonable approximation, by regular lattices [8]. Branching architectures, or coarse-grained models of compartimentalized units, justify invoking the generalized notion of a spatial network. In this case, the nodes stand for individual cellular units, linked via a heterogeneous web of intertangled connections, as exemplified by the network structure. The study of pattern formation for reaction-diffusion systems anchored on symmetric networks was developed in [13]. Diffusion instigates a spatial segregation of species, a counterintuitive outcome of Turing analysis, which holds true both in its continuous and discrete (lattice or network based) versions.

The standard Turing model assumes pattern arises from an homogeneous unpatterned state. However, as himself recognised this is not biologically realistic as "Most of the organism, most of the time is developing from one pattern into another, rather then from homogeneity to a pattern" [6]. One such pattern is asymmetry in flows, as displayed in real systems via chemical or electrical gradients [14]. For example, osmosis [14, 15] in the cell membrane or chemotaxis in the motility of cells $[8,16]$ are examples of asymmetric transport [17]. The case of Dictyostelium is also worth mentioning: this is a multicelled eukaryotic bacterivore, which develops pseudopodia under externally induced chemotaxis, so triggering 
a directed biased motion $[16,18]$. Rovinsky \& Menzinger $[19,20]$ proved that an inbalance in the directions of the flows of the species can indeed destabilise the spatially homogeneous state of the system. The differentialflow-induced instability yields oscillatory patterns for a sufficiently pronounced degree of asymmetry. It is, however, interesting to note that Othmer and Scriven had already intuited in their pioneering work [12] that asymmetric diffusion can eventually yield a richer pattern formation dynamics. Turing theory for systems evolving on directed networks was recently cast on rigorous grounds in [21] and asymmetry in the diffusion shown to produce a consistent enlargement of the region of Turing-like patterning.

Starting from these developments, we here take one important step forward in the aim of understanding the innate drive to self-organization as displayed by natural systems. We shall prove in particular that asymmetry in the transport across a - sufficiently large - multi-cellular (hence inherently discrete) compartment allows for patterns to be established, in simple reaction schemes and over an extended region of parameters values. Patterns display hence an unprecedented robustness, which ultimately stems from the parcelization of the embedding space in individual units of non-vanishing size. To anticipate some of the technical aspects to be addressed in the following, we will show that tuning the diffusivity of just one species - the ratio of the two diffusivities being frozen to a constant strictly different from (but arbitrarily close to) one - amounts to performing a homothetic transformation of the spectrum of the generalized Laplacian operator in the complex plane. The Laplacian eigenvalues consequently move along straight lines, the slope being set by the number, $\Omega$, of cells that define the multicellular array. By modulating these latter quantities, one can always get the eigenvalues to protrude into the region of instability. To allow for analytical progress to be made, we shall assume periodic boundary conditions, resulting from the rotational symmetry of the spatial domain. The patterns that follow the onset of the instability are either oscillatory or stationary. A pictorial representation of the proposed scheme is provided in panels $c$ ) and $d$ ) of Figure 1. Panels $a$ ) and $b$ ) refer instead to the conventional scenario which assumes undirected transport.

\section{THEORY OF PATTERN FORMATION WITH ASYMMETRIC DIFFUSION}

Let us begin by considering a generic two species model of reaction-diffusion type. The concentration of the two species are, respectively, labelled $u_{i}$ and $v_{i}$, where the index $i$ refers to the hosting cell and $i$ runs from 1 to $\Omega$.
The governing equations can be cast in the form:

$$
\begin{aligned}
& \frac{d u_{i}}{d t}=f\left(u_{i}, v_{i}\right)+D_{u}\left[\sum_{j=1}^{\Omega} A_{i j} u_{j}-\sum_{j=1}^{\Omega} A_{j i} u_{i}\right] \\
& \frac{d v_{i}}{d t}=g\left(u_{i}, v_{i}\right)+D_{v}\left[\sum_{j=1}^{\Omega} A_{i j} v_{j}-\sum_{j=1}^{\Omega} A_{j i} v_{i}\right]
\end{aligned}
$$

where $D_{u}$ and $D_{v}$ stand for the diffusion constants and $f(\cdot, \cdot), g(\cdot, \cdot)$ are the nonlinear reaction terms that model the local (on site) dynamics of the species. $A_{i j}$ are the binary entries of the adjacency matrix that specifies the topology of the embedding spatial support. For the case at hand we will focus on the limiting condition where species are solely allowed to jump clockwise, from a given cell to its immediate neighbor (panel $c$ ) of Fig. 1). Hence the adjacency matrix $A=\left\{A_{i j}\right\}$ is circulant (i.e. invariant under rotation) and the transport terms in square brackets in eqs. (1), take the form $u_{i-1}-u_{i}$ and $v_{i-1}-v_{i}$, respectively. Nevertheless, the results obtained here are valid in the general setting where the probability of hopping in the anti-clockwise direction is allowed for (see the Appendix 2). Although we shall be mainly interested in lattice arrangements, we emphasise that the transport operator introduced here represents the straightforward generalization of standard diffusion to the case where the spatial arrangements of the cells is supposed heterogeneous, either in terms of physical links, connecting individual patches of the collection, or in terms of their associated weights. Both are viable strategies for imposing the asymmetry which sits at the core of the mechanism upon which we shall hereby elaborate. In short, the hypothesized spatial coupling implements a local balance of incoming and outgoing fluxes, as seen from the observation cell $i$. We introduce the Laplacian operator $\mathbf{L}$ with entries $L_{i j}=A_{i j}-k_{i}^{\text {out }} \delta_{i j}$ where $\delta_{i j}$ denotes the Kronecker function and $k_{i}^{\text {out }}=\sum_{j=1}^{\Omega} A_{j i}$ quantifies the outgoing degree of cell $i$. The contributions that relate to inter-cell couplings in Eqs. (1) can be, respectively, rewritten in the equivalent, more compact form, $\sum_{j=1}^{\Omega} D_{u} L_{i j} u_{j}$ and $\sigma \sum_{j=1}^{\Omega} D_{u} L_{i j} v_{j}$, where $\sigma$ represents the ratio of the diffusion constants $D_{u}$ and $D_{v}$. In the following, we will denote with the symbol $\mathcal{L}$ the Laplacian $\mathbf{L}$, modulated by the multiplicative constant $D_{u}$, namely $\mathcal{L} \equiv D_{u} \mathbf{L}$. As a key observation for what follows, we emphasize that the magnitude of the eigenvalues of $\mathcal{L}$ can be freely controlled by the value of $D_{u}$. Changing $D_{u}$ (while for instance keeping $\sigma$ frozen) implies performing a homothetic transformation of the spectrum of $\mathbf{L}$, as we shall hereafter clarify. Notice that the problem is formulated at the discrete level and no attempt is made to eventually recover a continuous description of the examined dynamics. This is to reflect the fact that real tissue is composed of a large collection of individual cells. By increasing the number of cells in the lattice, we formally make the system larger, since the characteristic size of the cells cannot be contextually reduced. In practical 
a)

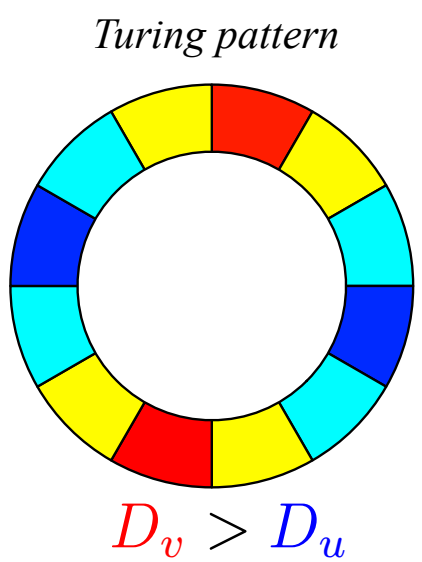

c)

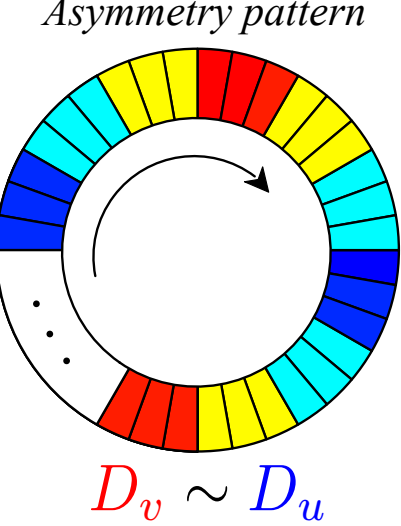

b)
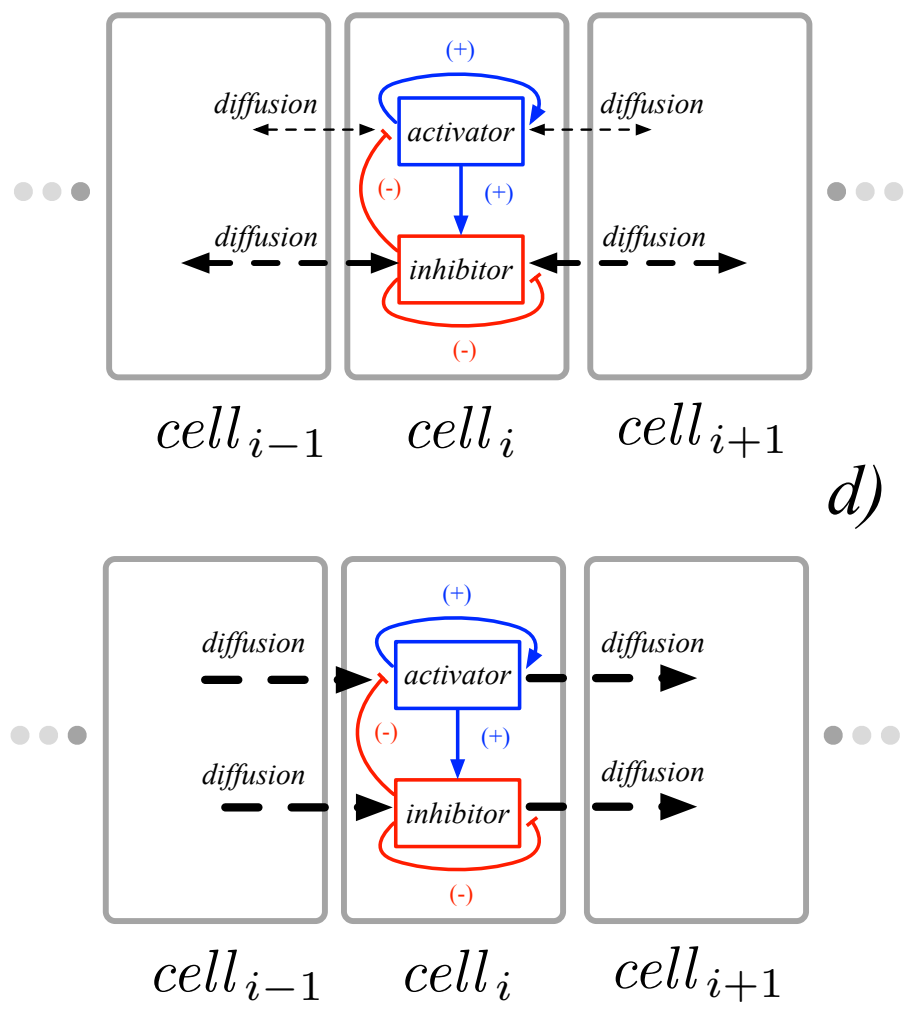

FIG. 1. Conventional Turing instability vs. the asymmetry-driven model: a schematic representation. (a) In Turing's original model, the onset of pattern formation is studied for a model of two species reacting and diffusing on a collection of cells, arranged so as to form a $1 D$ ring. (b) Turing instability requires breaking the symmetry among the species. In particular, feedback loops (positive for the activators and negative for the inhibitors) are proposed. Further, the inhibitor should relocate in space faster than the activator, $D_{v}>D_{u}$. The diffusion between neighboring cells is assumed symmetric. (c) In the asymmetry-induced instability instead the system is made up of a larger number of cells and the diffusion is asymmetric, as schematised by the clockwise arrow. (d) The instability is triggered by increasing the number of cells, for virtually any ratio of the diffusion constants, provided the latter take sufficiently large values.

terms, we will argue that the transition to the patterning regime can take place if the system reaches a critical size, as epitomized by the number of cells that compose its domain of action.

Assume now that the reaction dynamics admits a stable fixed point $\left(u^{*}, v^{*}\right)$, namely that $f\left(u^{*}, v^{*}\right)=$ $g\left(u^{*}, v^{*}\right)=0$. Hence, the spatially extended system (1) possesses a homogeneous equilibrium solution $\left(\mathbf{u}^{*}, \mathbf{v}^{*}\right)$ which is obtained by setting the variable on each of the $\Omega$ cells to be $\left(u^{*}, v^{*}\right)$. This conclusion can be readily derived by noticing that, by definition, $\sigma \sum_{j=1}^{\Omega} \mathcal{L}_{i j} K=0$, for any constant $K$ (recall that the system is hosted on a lattice with periodic boundary conditions). The homogeneous fixed point can, in principle, become unstable upon injection of a tiny heterogeneous perturbation, as in the spirit of the original Turing mechanism. The conditions for the emergence of the instability are determined by a linear stability analysis that follows the procedure pre- sented in [21] and that we revisit in the following.

\section{A. The dispersion relation and the conditions for instability}

Linearising the dynamics of system (1) around the fixed point solution $\left(\mathbf{u}^{*}, \mathbf{v}^{*}\right)$ we obtain:

$$
\begin{aligned}
\frac{d}{d t}\left(\begin{array}{l}
\delta \mathbf{u} \\
\delta \mathbf{v}
\end{array}\right) & =\left(\begin{array}{cc}
f_{u} \mathcal{I}+D_{u} \mathbf{L} & f_{v} \mathcal{I} \\
g_{u} \mathcal{I} & g_{v}+D_{v} \mathbf{L}
\end{array}\right)\left(\begin{array}{l}
\delta \mathbf{u} \\
\delta \mathbf{v}
\end{array}\right) \\
& =(\mathcal{J}+\mathcal{D})\left(\begin{array}{l}
\delta \mathbf{u} \\
\delta \mathbf{v}
\end{array}\right)
\end{aligned}
$$

where $\delta \mathbf{u}, \delta \mathbf{v}$ stands for the perturbation vectors. $\mathcal{J}=$ $\left(\begin{array}{ll}f_{u} \mathcal{I} & f_{v} \mathcal{I} \\ g_{u} \mathcal{I} & g_{v} \mathcal{I}\end{array}\right)$ is the Jacobian matrix, evaluated at the equilibrium point, stemming from the reaction terms and 
$\mathcal{D}=\left(\begin{array}{cc}D_{u} \mathbf{L} & \mathcal{O} \\ \mathcal{O} & D_{v} \mathbf{L}\end{array}\right)$, where $\mathcal{O}$ is the $\Omega \times \Omega$ zero-valued matrix. Introduce the basis formed by the eigenvectors $\Phi_{i}^{(\alpha)}$, with $\alpha=1, \ldots, \Omega$, of the Laplacian operator $\mathbf{L}$. We have $\sum_{j} L_{i j} \Phi_{j}^{(\alpha)}=\Lambda^{(\alpha)} \Phi_{i}^{(\alpha)}$ where $\Lambda^{(\alpha)}$ identifies the eigenvalues of $\mathbf{L}$. The latter operator is asymmetric, as is the matrix $\mathbf{A}$, and thus $\Lambda^{(\alpha)}$ are, in principle, complex. Further, the eigenvectors form an orthonormal basis, for the case at hand. Hence, to solve the above linear system, we expand the perturbation in terms of the basis of the eigenvectors, i.e. $\delta u_{i}=\sum_{\alpha=1}^{\Omega} b_{\alpha} \Phi_{i}^{(\alpha)}$ and $\delta v_{i}=\sum_{\alpha=1}^{\Omega} c_{\alpha} \Phi_{i}^{(\alpha)}$. At this point it is straightforward to show that the $2 \Omega \times 2 \Omega$ system reduces to a $2 \times 2$ eigenvalue problem, for each choice of the scalar index $\alpha=1, \ldots, \Omega$ :

$$
\begin{aligned}
\operatorname{det}\left(\mathbf{J}_{\alpha}-\lambda_{\alpha} \mathbf{I}\right) & =\operatorname{det}\left(\begin{array}{cc}
f_{u}+D_{u} \Lambda^{(\alpha)}-\lambda_{\alpha} & f_{v} \\
g_{u} & g_{v}+D_{v} \Lambda^{(\alpha)}-\lambda_{\alpha}
\end{array}\right) \\
& =0
\end{aligned}
$$

where $\mathbf{J}_{\alpha}$ is the $2 \times 2$ modified Jacobian, i.e. $\mathbf{J}_{\alpha} \equiv \mathbf{J}+$ $\mathbf{D} \Lambda^{(\alpha)}$ with $\mathbf{D}=\operatorname{diag}\left(D_{u}, D_{v}\right), \boldsymbol{\Lambda}^{(\alpha)}=\operatorname{diag}\left(\Lambda^{(\alpha)}, \Lambda^{(\alpha)}\right)$. The steady state is unstable to small heterogeneous perturbations, if $\lambda_{\alpha}$ has a positive real part over a finite range of modes. The dispersion relation (the largest real part of $\lambda_{\alpha}$, for any given $\alpha$ ) can be readily computed from:

$$
\lambda_{\alpha}=\frac{1}{2}\left[\left(\operatorname{tr} \mathbf{J}_{\alpha}\right)_{R e}+\gamma\right]+\frac{1}{2}\left[\left(\operatorname{tr} \mathbf{J}_{\alpha}\right)_{I m}+\delta\right] \iota
$$

where

$$
\begin{aligned}
& \gamma=\sqrt{\frac{A+\sqrt{A^{2}+B^{2}}}{2}} \\
& \delta=\operatorname{sgn}(B) \sqrt{\frac{-A+\sqrt{A^{2}+B^{2}}}{2}}
\end{aligned}
$$

and

$$
\begin{aligned}
& A=\left[\left(\operatorname{tr} \mathbf{J}_{\alpha}\right)_{R e}\right]^{2}-\left[\left(\operatorname{tr} \mathbf{J}_{\alpha}\right)_{I m}\right]^{2}-\left[\left(\operatorname{det} \mathbf{J}_{\alpha}\right)_{R e}\right]^{2} \\
& B=2\left(\operatorname{tr} \mathbf{J}_{\alpha}\right)_{R e}\left(\operatorname{tr} \mathbf{J}_{\alpha}\right)_{I m}-\left[\left(\operatorname{det} \mathbf{J}_{\alpha}\right)_{I m}\right]^{2} .
\end{aligned}
$$

Here $\operatorname{sgn}(\cdot)$ is the sign function and $f_{R e}, f_{I m}$ indicate, respectively, the real and imaginary parts of the operator $f$. To further manipulate Eq. (2), we make use of the definition of a square root of a complex number. Take, $z=a+b \iota$ where $\iota=\sqrt{-1}$ is the imaginary unit, then

$$
\sqrt{z}= \pm\left(\sqrt{\frac{a+|z|}{2}}+\operatorname{sgn}(b) \sqrt{\frac{-a+|z|}{2} \iota}\right) .
$$

The instability sets in when $\left|\left(\operatorname{tr} \mathbf{J}_{\alpha}\right)_{R e}\right| \leq \gamma$, a condition that translates into the following inequality [21]:

$$
S_{2}\left(\Lambda_{R e}^{(\alpha)}\right)\left[\Lambda_{I m}^{(\alpha)}\right]^{2} \leq-S_{1}\left(\Lambda_{R e}^{(\alpha)}\right)
$$

where $\left(\Lambda_{R e}, \Lambda_{I m}\right)$ span the complex plane where the Laplacian eigenvalues reside. In the above relation $S_{1}, S_{2}$ are polynomials which take the following explicit form:

$$
\begin{aligned}
& S_{1}(x)=C_{14} x^{4}+C_{13} x^{3}+C_{12} x^{2}+C_{11} x+C_{10} \\
& S_{2}(x)=C_{22} x^{2}+C_{21} x+C_{20} .
\end{aligned}
$$

The constants here are given by:

$$
\begin{aligned}
& C_{14}=\sigma(1+\sigma)^{2} \\
& C_{13}=(1+\sigma)^{2}\left(\sigma J_{11}+J_{22}\right)+2 \operatorname{tr} \mathbf{J} \sigma(1+\sigma) \\
& C_{12}=\operatorname{det} \mathbf{J}(1+\sigma)^{2}+(\operatorname{tr} \mathbf{J})^{2} \sigma+2 \operatorname{tr} \mathbf{J}(1+\sigma)\left(\sigma J_{11}+J_{22}\right) \\
& C_{11}=2 \operatorname{tr} \mathbf{J}(1+\sigma)^{2} \operatorname{det} \mathbf{J}+(\operatorname{tr} \mathbf{J})^{2}\left(\sigma J_{11}+J_{22}\right) \\
& C_{10}=\operatorname{det} \mathbf{J}(\operatorname{tr} \mathbf{J})^{2}
\end{aligned}
$$

and

$$
\begin{aligned}
& C_{22}=\sigma(1-\sigma)^{2} \\
& C_{21}=\left(\sigma J_{11}+J_{22}\right)(1-\sigma)^{2} \\
& C_{20}=J_{11} J_{22}(1-\sigma)^{2} .
\end{aligned}
$$

The system displays a generalized Turing instability, determined by the asymmetric nature of the imposed coupling if, after the homothetic transformation, the eigenvalues $D_{u}\left(\Lambda_{R e}^{(\alpha)}, \Lambda_{I m}^{(\alpha)}\right)$ of the Laplacian operator $\mathcal{L}$ enter the region of the complex plane $\left(\Lambda_{R e}, \Lambda_{I m}\right)$ that is delimited by inequality (3). Notice that the instability region is drawn by taking $D_{u} \Lambda_{R e}^{(\alpha)} \equiv \Lambda_{R e}$ and $D_{u} \Lambda_{I m}^{(\alpha)} \equiv \Lambda_{I m}$ in the complex plane $\left(\Lambda_{R e}, \Lambda_{I m}\right)$ such that it remains invariant to $D_{u}$ and the spectrum of $\mathcal{L}$.

The latter relation (3) can be graphically illustrated in the complex plane $\mathbf{z}=\left(\Lambda_{R e}, \Lambda_{I m}\right)$, where the eigenvalues $\Lambda^{(\alpha)}$ of the Laplacian operator $\mathbf{L}$ reside (see Appendix 1). In fact, inequality (3) enables one to delimit a model-dependent region of instability, which is depicted in panel $a$ ) of Fig. 2. When drawing the domain of interest, we assumed an abstract setting, without insisting on the specific details that stem from a particular reaction model. For what will follow, it is only important to appreciate that condition (3) makes it possible to define a portion of the parameter space, by construction symmetric with respect to the horizontal (real) axis, that is eventually associated with the onset of the instability. More specifically, if a subset of the spectrum of the Laplacian $\mathcal{L}$ falls inside the region outlined above, the instability can take place (red stars in panel $a$ ) of Fig. 2). This conclusion is general and independent of the reaction scheme employed. Notice that when the two regions merge and incorporate a finite part of the real axis, the outbreak of the instability is also possible on a symmetric support (i.e. when the eigenvalues of the Laplacian operator are real).

\section{UNIVERSAL MECHANISM FOR PATTERN FORMATION}

We are now in a position to elaborate on the universal mechanism which drives a reaction-transport system 

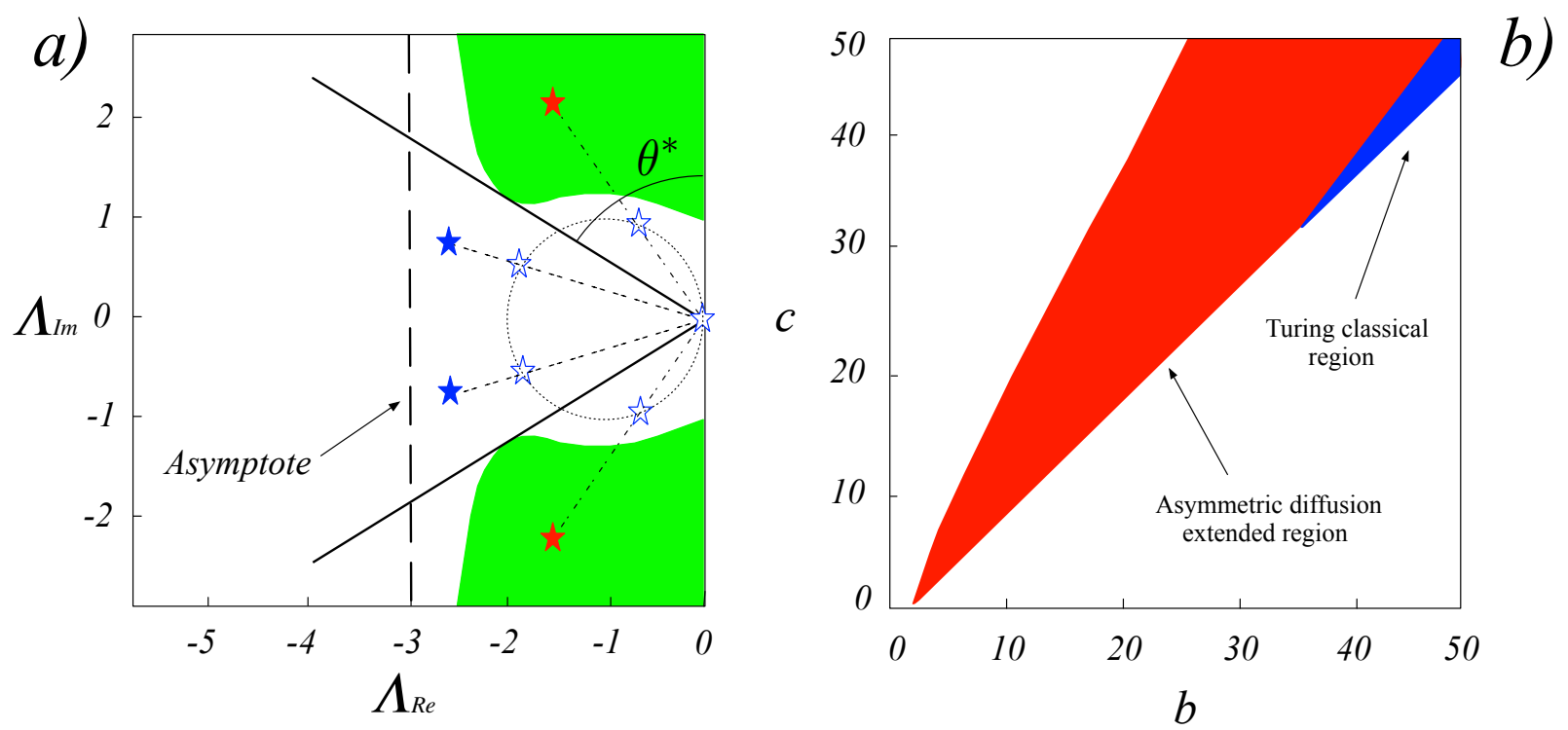

FIG. 2. On the mechanism of pattern formation with asymmetric diffusion. a) The region colored in green denotes the domain of the complex plane $\mathbf{z}=\left(\Lambda_{R e}, \Lambda_{I m}\right)$ where the instability can eventually take place. This is a pictorial representation of a general situation that is always found, irrespectively of the specific choice of the reaction model. The blue empty symbols stand for the spectrum of the Laplacian operator $\mathbf{L}$ obtained from a directed lattice of the type depicted in Fig. $1 \mathrm{c}$ ). The eigenvalues are distributed on the unitary circle, as discussed in the Methods section. When increasing $\Omega$, the number of cells that compose the examined lattice, the Laplacian spectrum displays eigenvalues with progressively larger (but still negative) component $\Lambda_{R e}$ and still lying on the circle. We denote by $\theta$ the inclination of the line (dot dashed in the figure) that connects the selected eigenvalue (empty star) to the origin of the complex plane. This latter eigenvalue can be freely moved along the aforementioned line, by modulating the diffusion coefficient $D_{u}$ while keeping the ratio $\sigma$ fixed. Stated differently, the spectrum of the matrix $\mathcal{L}$ is an homothetic transformation (with centre 0 and ratio $D_{u}$ ) of the spectrum of $\mathbf{L}$. If $\theta$ is sufficiently small, i.e. if the dashed line is steeper than the solid one (the instability threshold), it is always possible (by increasing $D_{u}$ ) to move the associated eigenvalue inside the region (green) of the instability (see the filled red star). If the inclination $\theta$ is larger than the critical one, $\theta^{*}$, the eigenvalues that slide on the corresponding dashed line are permanently confined outside the domain of instability (filled blue star). The vertical dashed line is an asymptote and sets the leftmost boundary of the instability domain, as described in the Appendix 1. b) To provide a quantitative illustration of the method, we consider the Brusselator model, $f(u, v)=1-(b+1) u+c u^{2} v$ and $g(u, v)=b u-c u^{2} v$, where $b$ and $c$ are free control parameters. In the plane $(b, c)$, we isolate the domain where the conventional Turing instability takes place (small blue domain), for $\sigma=1.4$. The region shaded in red identifies the domain of instability that is found when the system is evolved on a directed lattice made of $\Omega=1000$ cells and assuming $D_{u}=100$. The ratio $\sigma$ is kept constant to the reference value 1.4.

unstable, for asymmetric diffusion on a discrete collection of lattice sites. As we shall realize, considering a finite, although large, ensemble of mutually connected cells is one of the key ingredients that instigates the instability in an activator-inhibitor system for virtually any reaction parameters and any ratio of the diffusion coefficients (including $\sigma<1$ and except for the zero measure, limiting condition $\sigma=1$ ). We begin by observing that the Laplacian matrix $\mathbf{L}$, associated with a closed directed ring as assumed in Fig. $1 \mathrm{c}$ ), is a circulant matrix. This simplified geometrical arrangement is solely assumed for illustrative purposes. A $\Omega \times \Omega$ matrix $C$ is circulant if it takes the form

$$
\mathbf{C}=\left(\begin{array}{ccccc}
c_{0} & c_{\Omega-1} & \ldots & c_{2} & c_{1} \\
c_{1} & c_{0} & c_{\Omega-1} & \ldots & c_{2} \\
\vdots & c_{1} & c_{0} & \ddots & \vdots \\
c_{\Omega-2} & & \ddots & \ddots & c_{\Omega-1} \\
c_{\Omega-1} & c_{\Omega-2} & \ldots & c_{1} & c_{0}
\end{array}\right)
$$

The circulant matrix $\mathbf{C}$ is fully specified by its first column, $\mathbf{c}=\left(c_{0}, c_{1}, \ldots c_{\Omega-1}\right)$. The other columns of C are generated as cyclic permutations of the vector c with offset equal to the column index. The normalized eigenvectors of a circulant matrix are given by $\phi_{j}=\frac{1}{\sqrt{\Omega}}\left(1, \omega_{j}, \omega_{j}^{2}, \ldots, \omega_{j}^{\Omega-1}\right)$ where $\omega_{j}=\exp (2 \pi \iota j / \Omega)$ is the $\Omega$-th root of the unity and the eigenvalues are $\lambda_{j}=c_{0}+c_{\Omega-1} \omega_{j}+c_{\Omega-2} \omega_{j}^{2}+\cdots+c_{1} \omega_{j}^{\Omega-1}$ where $j=$ $0,1, \ldots, \Omega-1$. Hence the spectrum of $\mathbf{L}$ is complex and falls on the unitary circle centered at $(-1,0)$ - empty stars in Fig. 2, panel $a$ ).

When making $\Omega$ larger, one progressively reduces the spectral gap, the relative distance between the two Laplacian eigenvalues that display the largest real parts. Recalling that the largest eigenvalue of the Laplacian is by definition zero, this implies that the second eigenvalue of $\mathbf{L}$ (ranked in descending order, with respect to the value of their associated real parts) tends to approach the origin of the complex plane, when $\Omega$ is increased [22]. This, 

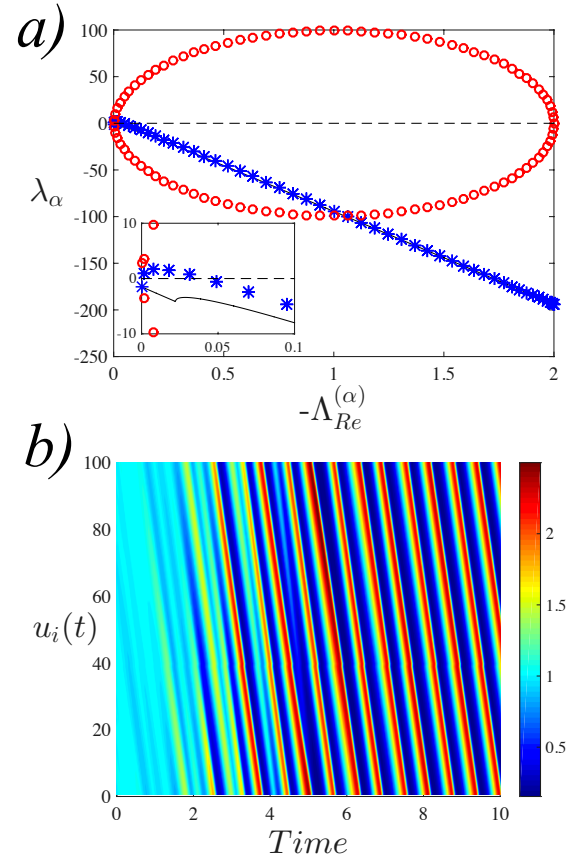
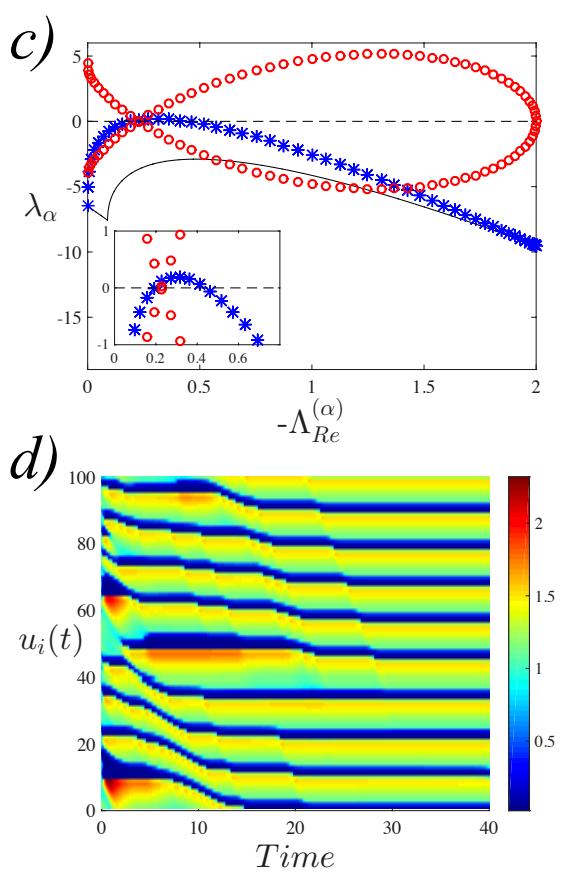
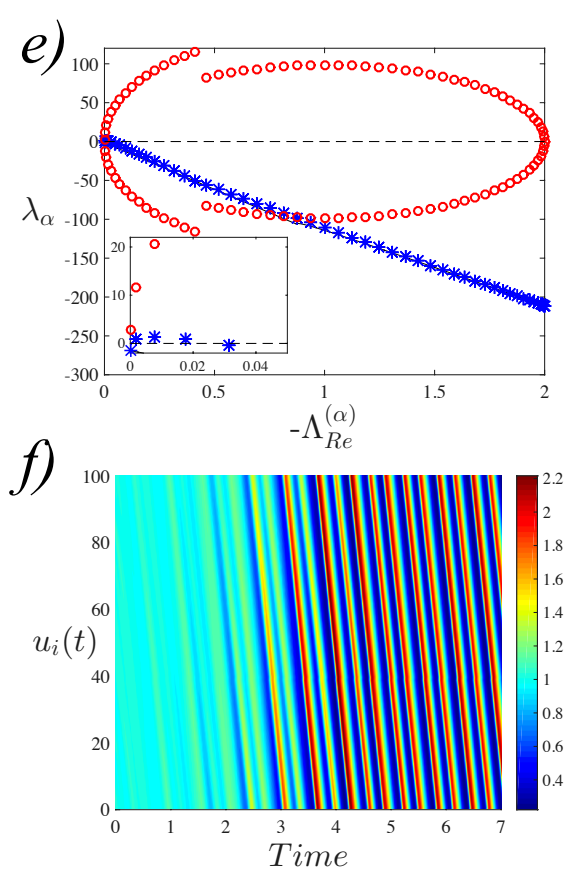

FIG. 3. Patterns triggered in the Brusselator model by asymmetric diffusion on a $1 D$ ring. Left column: real (blue stars) and imaginary (red circles) dispersion relation $\lambda_{\alpha} \mathbf{a}$ ) and the associated pattern evolution $\mathbf{b}$ ). The ensuing pattern is oscillatory and organizes as a travelling wave. The parameters are set to $b=8, c=10, D_{u}=100$, and $\sigma=1.4$. Center column: dispersion relation c) and pattern evolution d) yielding a steady state pattern, when $b=50, c=62, D_{u}=10$ and $\sigma=1.4$. Right column: dispersion relation e) and pattern evolution f) yielding a traveling wave with $b=8, c=10, D_{u}=140$ and $\sigma=1 / 1.4 \simeq 0.71$. In the insets in the upper panels, we zoom on the (real and imaginary) dispersion relations focusing on the portion of the curve where the instability takes place. In all cases, $\Omega=100$. Notice that, for the Brusselator model, oscillatory and stationary patterns are numerically detected for $\sigma>1$, while for $\sigma<1$ oscillatory patterns are solely found. There is, however, no reason to exclude that other reaction schemes would yield stationary stable patterns for $\sigma<1$ as an outcome of the scheme shown here.

in turn, implies that we can control at will the inclination (a measure complementary to the slope) $\theta$ of the line (dashed in the figure) that connects the second largest eigenvalue to the origin of the complex plane. Similar considerations apply to the other eigenvalues that follow in the ranking. In Fig. 2) a) we label by $\theta^{*}$ the critical inclination of the solid line that intersects tangentially the domain of instability traced according to inequality (3). Clearly, by construction, a symmetric line always exists with inclination $-\theta^{*}$, that is tangent to the region of instability in the lower complex semi-plane. Assume now that $\Omega$ is sufficiently large so that $\theta<\theta^{*}$. Then recall that for the instability to emerge, at least one eigenvalue of the rescaled Laplacian $\mathcal{L}$ has to protrude into the region where the instability is bound to occur. On the other hand, the eigenvalues of $\mathcal{L}$ are a homothetic transformation, with centre 0 and ratio $D_{u}$, of the eigenvalues $\mathbf{L}$. In other words, we can force the second eigenvalue of $\mathbf{L}$ to move along the line to which it belongs by modulating the diffusion constant $D_{u}$, while keeping $\sigma$ constant, and so invade the region of the instability (filled red star). If $\theta>\theta^{*}$, then the eigenvalue is instead confined to the stable portion of the complex plane for each choice of the scaling factor $D_{u}$. In summary, the system can be triggered unstable by simultaneously acting on two indepen- dents "knobs": first, by making $\Omega$ sufficiently large, we force a subset of eigenvalues into the vicinity of the origin, thus making their associated $\theta$ smaller that the critical amount $\theta^{*}$. Building on the analysis performed in the Appendix, see Eq. (17), the above condition materializes in $\Omega>2 \pi /\left(\arctan f^{\prime}\left(r_{0}\right)-\pi / 2\right)$, where the prime denote the first derivative of $f(x)=\sqrt{-S_{1}(x) / S_{2}(x)}$ and $r_{0}$ is the abscissa of the point where the line passing through the origin intersects tangentially the curve $f=0$. Secondly, by acting on $D_{u}$ (and consequently on $D_{v}$ so as to maintain $\sigma$ unchanged), we make the eigenvalues slide on their corresponding lines, until the instability threshold is eventually breached. Interestingly, the instability involves long wavelengths (hence yielding macroscopic patterns) since, by construction, the real part of the eigenvalues associated with the modes (eigenvectors) triggered to grow approaches zero, when $\Omega$ increases.

In Fig. 2 b) we present results for a specific case study, the Brusselator model, often invoked in the literature as a paradigm nonlinear reaction scheme for studying selforganised phenomena, synchronisation, Turing patterns and oscillation death. We find the region in the parameter space $(b, c)$ for which the homogeneous steady state is unstable for $\sigma=1.4$. The Brusselator may undergo 
a conventional Turing instability, in the portion of the plane that is colored in blue. The asymmetry driven instability (for $\Omega=1000$ cells and assuming $D_{u}=100$ ) is found on a considerably larger domain, which can be made arbitrarily large by further modulating $\Omega$ and $D_{u}$.

In Fig. 3, panels $b$ ), $d$ ) and $f$ ), we show patterns found when integrating the Brusselator model for different parameter values. Panels $a$ ), $c$ ) and $e$ ) display the real and imaginary parts of the eigenvalues $\lambda_{\alpha}$ (the dispersion relation, see above) against $-\Lambda_{R e}^{(\alpha)}$. When the imaginary component of $\lambda_{\alpha}$ is small as compared to the corresponding real part, steady state patterns are found to emerge. Otherwise, the patterns are oscillatory in nature. This is a rule of the thumb, which seems to implement a necessary criterion. The mechanism of pattern selection is in fact heavily influenced by the nonlinearities, which become prominent beyond the initial stages of evolution, hence limiting the predictive ability of the linear stability analysis. It is worth stressing that the patterns displayed in Fig. 3 occur for a choice of parameters for which the classical Turing instability is not permitted and for both $\sigma$ larger and smaller than one. In particular, one can analytically show that the asymmetry driven instability, that we here introduced and characterized, can develop for any $\sigma=1 \pm \epsilon$ with $0<\epsilon$. This conclusion holds in general, as can be proved rigorously (see Appendix 1).

\section{DISCUSSION}

To summarise, we have developed a general theory of pattern formation for the case of asymmetric transport of constituents in a large assembly of adjacent cells. Macroscopic oscillatory or steady state patterns in an activatorinhibitor system are now found for virtually any ratio of diffusivities, larger or smaller than one, and any choice of the reaction parameters. The system (as composed by a large ensemble of individual units, the cells) should extend in space beyond a critical size for the instability to eventually develop, at a chosen ratio of the diffusivities. It is worth stressing that this observation might be relevant to embryogenesis applications. In fact, it is known that cell specialization for tissue development starts only when the system has attained a critical size, resulting from the aggregation of sufficiently many embryonic cells [23]. In our theory we deal, for simplicity, with a one-dimensional arrangement of cells which define the spatial support to the model. The one-dimensional cell lattice has periodic boundary conditions. The analysis can be in principle extended to higher dimensions, by performing a Cartesian product of fundamental onedimensional units of the type analyzed here [24]. Periodic boundary conditions in extended spatial dimensions, can be for instance invoked when mimicking organs in their embryonic stage $[25,26]$ with tubular or globular shapes. Our conclusion holds however more generally, also when the assumption of dealing with periodic boundary conditions (which make the problem analytically tractable) is eventually relaxed [27]. Taken all together, the potential applications of this newly proposed route to pattern formation are multiple from organogenesis to embryonic development and may also include all those settings, from biology to ecology passing through neuroscience [14-16, 18, 28, 29], where asymmetric flows are reported to occur.

\section{APPENDIX}

\section{On the shape of the instability domain and the onset of the directed instability}

The aims of this section are twofold. We will first provide a detailed characterization of the region $\mathcal{D}$ of the complex plane $\left(\Lambda_{R e}, \Lambda_{I m}\right)$ where the instability eventually takes place. Then, we will show that a non-zero inclination $\theta^{*}$ exists for any value of $\sigma$ strictly different from one. More specifically, we shall prove that the line tangent to the instability domain displays a slope which scales as $1 / \epsilon$, for $\sigma=1 \pm \epsilon$ and $0<\epsilon \ll 1$. This observation yields an important consequence. It is in fact always possible to select a minimal lattice size, $\Omega$, so as to have $\theta$, the inclination of the line that connects the second eigenvalue to the origin, smaller than $\theta^{*}>0$. This implies, in turn, that the system can be always made unstable by appropriately setting the strength of $D_{u}$ (and consequently $D_{v}$ ), while keeping $\sigma$ fixed to a nominal value arbitrarily close to one. The instability cannot be seeded at $\sigma=1\left(\theta^{*}\right.$, being in this case zero, or, equivalently the slope of the tangent to $\mathcal{D}$, infinite), a zero measure limiting condition.

By using a symbolic manipulator, it is possible to show that the fourth degree polynomial $S_{1}(x)$ admits a double root at $x_{1}=-\operatorname{tr} \mathbf{J} /\left(D_{v}+D_{u}\right)$, which is a positive defined number because of the necessary assumption $\operatorname{tr} \mathbf{J}<0$ (stability of the homogeneous fixed point). Factorising this latter term, we can write $S_{1}(x)=(x+\operatorname{tr} \mathbf{J})^{2} Q_{1}(x)$ where

$$
Q_{1}(x)=\left(D_{v}+D_{u}\right)\left(\operatorname{det} \mathbf{J}+\left(D_{u} J_{22}+D_{v} J_{11}\right) x+D_{u} D_{v} x^{2}\right)
$$

is a second order polynomial whose roots are straightforwardly determined as

$$
\begin{aligned}
& x_{3}=\frac{-\left(D_{u} J_{22}+D_{v} J_{11}\right)-\sqrt{\left(D_{u} J_{22}+D_{v} J_{11}\right)^{2}-4 D_{u} D_{v} \operatorname{det} \mathbf{J}}}{2 D_{u} D_{v}} \\
& x_{4}=\frac{-\left(D_{u} J_{22}+D_{v} J_{11}\right)+\sqrt{\left(D_{u} J_{22}+D_{v} J_{11}\right)^{2}-4 D_{u} D_{v} \operatorname{det} \mathbf{J}}}{2 D_{u} D_{v}} .
\end{aligned}
$$

If $\left(D_{u} J_{22}+D_{v} J_{11}\right)^{2}-4 D_{u} D_{v} \operatorname{det} \mathbf{J}>0$, then $x_{3}<x_{4}$; otherwise $Q_{1}(x)>0$ for all $x$. In fact, $\operatorname{det} \mathbf{J}>0$ owing to the stability of the homogeneous fixed point.

The characterization of $S_{2}(x)$ is straightforward as it 
is a second degree polynomial. Its roots can be written:

$$
y_{1}=-\frac{J_{22}}{D_{v}} \quad \text { and } y_{2}=-\frac{J_{11}}{D_{u}} .
$$

Let us observe that the assumption of a stable homogeneous equilibrium implies $\operatorname{tr} \mathbf{J}<0$. Thus, $y_{1}$ and $y_{2}$ cannot be both negative. On the other hand, relation (3) cannot be satisfied for negative $\Lambda_{R e}$, if $y_{1}$ and $y_{2}$ are both positive, as it readily follows from a direct computation. Without loss of generality, we can thus assume $J_{11}>0$ and $J_{22}<0$ (as for the case of the Brusselator model). Hence, the two coupled species should be necessarily linked via an activator-inhibitor scheme, for the generalized instability to eventually develop, as in the original Turing framework. Recall however that the route to instability here discussed enables one to virtually remove all constraints on the diffusion constants, as imposed under Turing's approach.

Assume thus, $y_{1}>0$ and $y_{2}<0$. Observe that when $S_{1}$ vanishes, the domain $\mathcal{D}$ hits the $x$-axis with a vertical slope (provided $S_{2}$ is non-zero at the same time, which is generically true). On the other hand, if $S_{2}$ vanishes (but not $S_{1}$, which is again true in general) then the domain displays a vertical asymptote (the vertical dashed line in Fig. 2 in the Main Text).

Collecting together the above information, we can isolate the following possible scenarios:

$\mathbf{A}:\left(D_{u} J_{22}+D_{v} J_{11}\right)^{2}-4 D_{u} D_{v} \operatorname{det} \mathbf{J}>0$ and $D_{u} J_{22}+$ $D_{v} J_{11}<0$ (see Fig. 4). Restricting the analysis to the relevant domain $x \leq 0, S_{1}(x)$ is always positive while $S_{2}(x)$ is positive for $x<y_{2}$ and negative otherwise. The domain $\mathcal{D}$ therefore displays a vertical asymptote at $x=y_{2}$.

B: $\left(D_{u} J_{22}+D_{v} J_{11}\right)^{2}-4 D_{u} D_{v} \operatorname{det} \mathbf{J}>0$ and $D_{u} J_{22}+$ $D_{v} J_{11}>0$. Here, we can identify three sub-cases, depending on the relative positions of the roots $x_{3}$, $x_{4}$ and $y_{2}$.

B.1: $x_{3}<x_{4}<y_{2}$ (see Fig. 5). In the negative half-plane the domain $\mathcal{D}$ is composed of the following parts: two unbounded regions never touching the $x$-axis and a bounded domain with a finite intersection with the $x$-axis; the latter can be responsible for the emergence of Turing patterns on symmetric supports. $S_{1}(x)$ changes sign twice while $S_{2}(x)$ is positive for $x<y_{2}$ and negative otherwise.

B.2: $x_{3}<y_{2}<x_{4}$ (see Fig. 6). In the negative half-plane $\mathcal{D}$ is composed of a single domain intersecting the $x$-axis. Hence Turing patterns on symmetric supports can develop. In the interval $\left(y_{2}, x_{4}\right)$ both $S_{1}(x)$ and $S_{2}(x)$ are negative, hence the inequality $S_{2}(x) y^{2} \leq-S_{1}(x)$ is satisfied for all $y$.

B.3: $y_{2}<x_{3}<x_{4}$ (see Fig. 7). In the negative half-plane $\mathcal{D}$ is composed again of a single domain intersecting the $x$-axis. Hence Turing patterns on symmetric support can possibly occur. In the interval $\left(x_{3}, x_{4}\right)$ both $S_{1}(x)$ and $S_{2}(x)$ are negative, hence the inequality $S_{2}(x) y^{2} \leq-S_{1}(x)$ is satisfied for all $y$.

C: $\left(D_{u} J_{22}+D_{v} J_{11}\right)^{2}-4 D_{u} D_{v} \operatorname{det} \mathbf{J}<0$ (see Fig. 8). $S_{1}(x)$ is always positive while $S_{2}(x)$ is positive for $x<y_{2}$ and negative otherwise in the negative halfplane $x \leq 0$. The domain $\mathcal{D}$ displays a vertical asymptote at $x=y_{2}$.

In the cases where Turing patterns cannot develop on a symmetric network, namely $\mathbf{A}$ and $\mathbf{C}$ (we will also consider case $\mathbf{B} .1$ because of its peculiar shape), we are interested in determining the straight line that passes through the origin and is tangent to the domain $\mathcal{D}$. Because the latter domain presents a clear symmetry $y \mapsto-y$ we will limit ourselves to considering the tangency condition for positive values of $y$. Further we shall denote by $y=f(x)=\sqrt{-S_{1}(x) / S_{2}(x)}$ the boundary of $\mathcal{D}$.

We select a generic point on the boundary of $\mathcal{D}$, that is, a point with coordinates $\left(x_{0}, f\left(x_{0}\right)\right.$ ) (see Fig. 9). Then, we consider the equation of the line tangent to the domain in $x_{0}$, i.e. $y=f^{\prime}\left(x_{0}\right)\left(x-x_{0}\right)+f\left(x_{0}\right)$. Further, we let $x_{0}$ vary and among all the straight lines, we select the one passing through the origin, i.e. setting $x=y=0$ in the previous equation. The coordinate of such a point must hence satisfy: $0=-x_{0} f^{\prime}\left(x_{0}\right)+f\left(x_{0}\right)$.

Using the definition of $f(x), x_{0}$ must satisfy

$$
2 S_{1}\left(x_{0}\right) S_{2}\left(x_{0}\right)-x_{0}\left(S_{1}^{\prime}\left(x_{0}\right) S_{2}\left(x_{0}\right)-S_{1}\left(x_{0}\right) S_{2}^{\prime}\left(x_{0}\right)=0\right.
$$

this appears to be a 6 -th order polynomial. It can be shown that the coefficient of $x_{0}^{6}$ is identically equal to zero because of the definition of $C_{i j}$. We therefore have a 5 -th order polynomial, that we label $P_{5}\left(x_{0}\right)$. By using a symbolic manipulator, we can show that $-\operatorname{tr} \mathbf{J} /\left(D_{v}+D_{u}\right)$ is a root of $P_{5}\left(x_{0}\right)$ [30]. We are hence finally left with $P_{5}\left(x_{0}\right)=\left(x_{0}+\operatorname{tr} \mathbf{J} /\left(D_{v}+D_{u}\right)\right) P_{4}\left(x_{0}\right)$, where $P_{4}\left(x_{0}\right)$ is a polynomial of 4 -th degree, whose roots can be analytically determined by using well known formulas.

To proceed in the analysis, we write $P_{4}\left(x_{0}\right)=e+d x_{0}+$ $c x_{0}^{2}+b x_{0}^{3}+a x_{0}^{4}$. Then the four roots read

$$
\begin{aligned}
& r_{1}=-\frac{b}{4 a}-S+\frac{1}{2} \sqrt{-4 S^{2}-2 p+\frac{q}{S}} \\
& r_{2}=-\frac{b}{4 a}-S-\frac{1}{2} \sqrt{-4 S^{2}-2 p+\frac{q}{S}} \\
& r_{3}=-\frac{b}{4 a}+S+\frac{1}{2} \sqrt{-4 S^{2}-2 p-\frac{q}{S}} \\
& r_{4}=-\frac{b}{4 a}+S-\frac{1}{2} \sqrt{-4 S^{2}-2 p-\frac{q}{S}}
\end{aligned}
$$



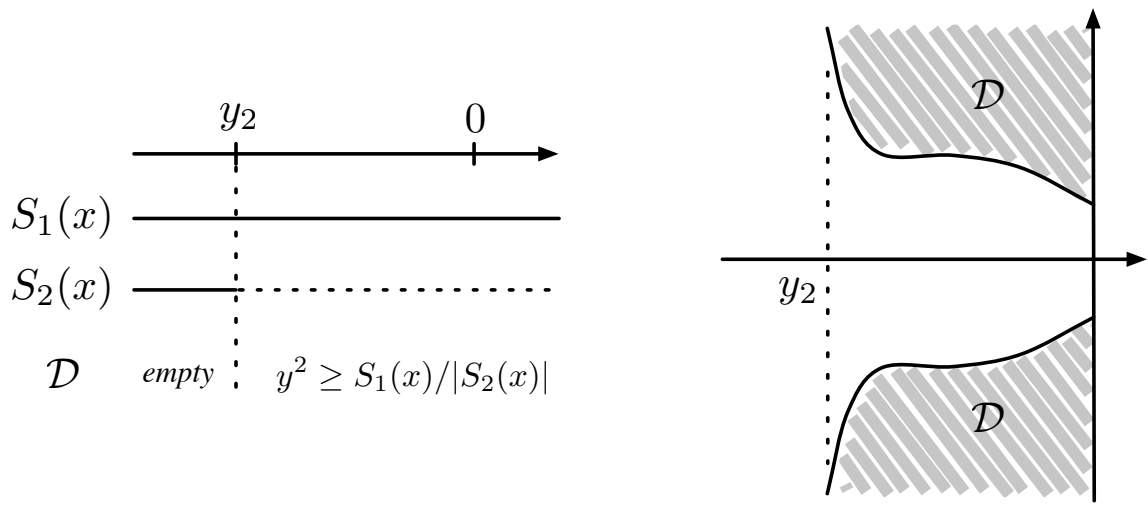

FIG. 4. The domain $\mathcal{D}$ : $\left(D_{u} J_{22}+D_{v} J_{11}\right)^{2}-4 D_{u} D_{v} \operatorname{det} \mathbf{J}>0$ and $D_{u} J_{22}+D_{v} J_{11}<0$. Left panel: the signs of the functions $S_{1}(x)$ and $S_{2}(x)$ for $x \leq 0$. Right panel: the corresponding domain $\mathcal{D}$ is depicted. Note the vertical asymptote and the fact that the domain has a limited extension on the horizontal axis. The domain never intercepts the $x$-axis. Hence, Turing patterns cannot develop on a symmetric support, whose Laplacian spectrum is real.
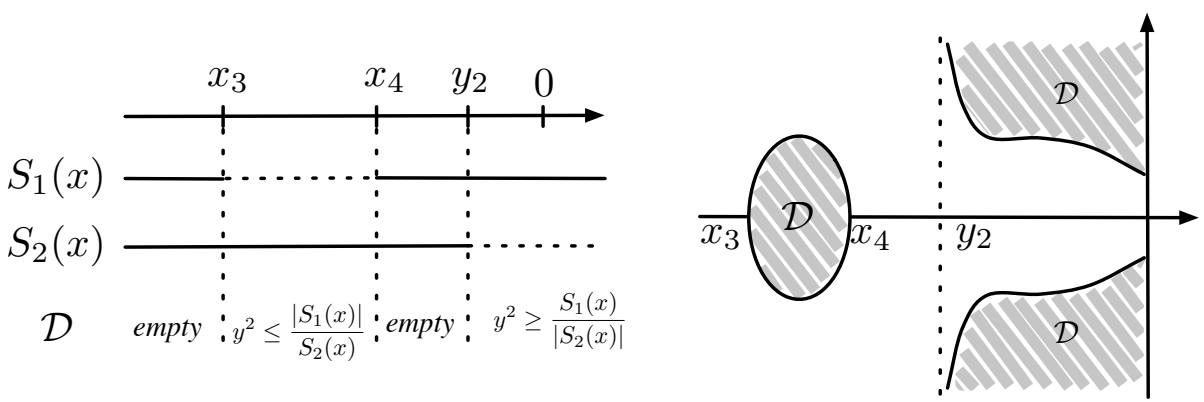

FIG. 5. The domain $\mathcal{D}:\left(D_{u} J_{22}+D_{v} J_{11}\right)^{2}-4 D_{u} D_{v} \operatorname{det} \mathbf{J}>0$ and $D_{u} J_{22}+D_{v} J_{11}>0, x_{3}<x_{4}<y_{2}$. Left panel: the signs of the functions $S_{1}(x)$ and $S_{2}(x)$ are characterized for $x \leq 0$. Right panel: the corresponding shape of the $\mathcal{D}$ domain is plotted. Note the vertical asymptote and the fact that the domain has a limited extension on the horizontal axis. The domain intercepts the $x$-axis over a finite interval. Turing patterns can hence develop on symmetric supports.

where

$$
\begin{aligned}
p & =\frac{8 a c-3 b^{2}}{8 a^{2}} \\
q & =\frac{b^{3}-4 a b c+8 a^{2} d}{8 a^{3}} \\
\Delta_{0} & =c^{2}-3 b d+12 a e \\
\Delta_{1} & =2 c^{3}-9 b c d+27 b^{2} e+27 a d^{2}-72 a c e \\
Q & =\left[\frac{\Delta_{1}+\sqrt{\Delta_{1}^{2}-4 \Delta_{0}^{3}}}{2}\right]^{1 / 3} \\
S & =\frac{1}{2} \sqrt{-\frac{2}{3} p+\frac{1}{3 a}\left(Q+\frac{\Delta_{0}}{Q}\right)} .
\end{aligned}
$$

Let us call $r_{0}$ any negative (real) solution. Then, we need to evaluate $f^{\prime}\left(r_{0}\right)$, the slope of the line passing through the origin and tangent to the domain $\mathcal{D}$. Let us observe that such value is related to the critical angle $\theta^{*}$, as defined in the main text (see Fig. 2), by the relation

$$
\theta^{*}=-\pi / 2+\arctan f^{\prime}\left(r_{0}\right)
$$

$f^{\prime}\left(r_{0}\right)$ will depend on the set of parameters involved. As anticipated in the beginning of this section, we aim at characterizing the dependence of $f^{\prime}\left(r_{0}\right)$ on $\epsilon$, when setting $\sigma=1 \pm \epsilon$ and for $\epsilon \ll 1$. To this end we observe that the coefficients $C_{2 i}$ can be rewritten as:

$$
\begin{aligned}
& C_{22}=D_{u}^{4}(1+\epsilon) \epsilon^{2}=\hat{C}_{22} \epsilon^{2} \\
& C_{21}=D_{u}^{3}\left((1+\epsilon) J_{11}+J_{22}\right) \epsilon^{2}=\hat{C}_{21} \epsilon^{2} \\
& C_{20}=J_{11} J_{22} D_{u}^{2} \epsilon^{2}=\hat{C}_{20} \epsilon^{2},
\end{aligned}
$$

where $\hat{C}_{2 i}$ are consistently defined by the above equalities. As a consequence, we can factor out a term $\epsilon^{2}$ from $S_{2}(x)$. This implies that $f(x)$ can be rewritten has

$$
f(x)=\sqrt{-\frac{S_{1}(x)}{S_{2}(x)}}=\frac{1}{\epsilon} \sqrt{-\frac{S_{1}(x)}{\hat{S}_{2}(x)}}=\frac{1}{\epsilon} \hat{f}(x),
$$



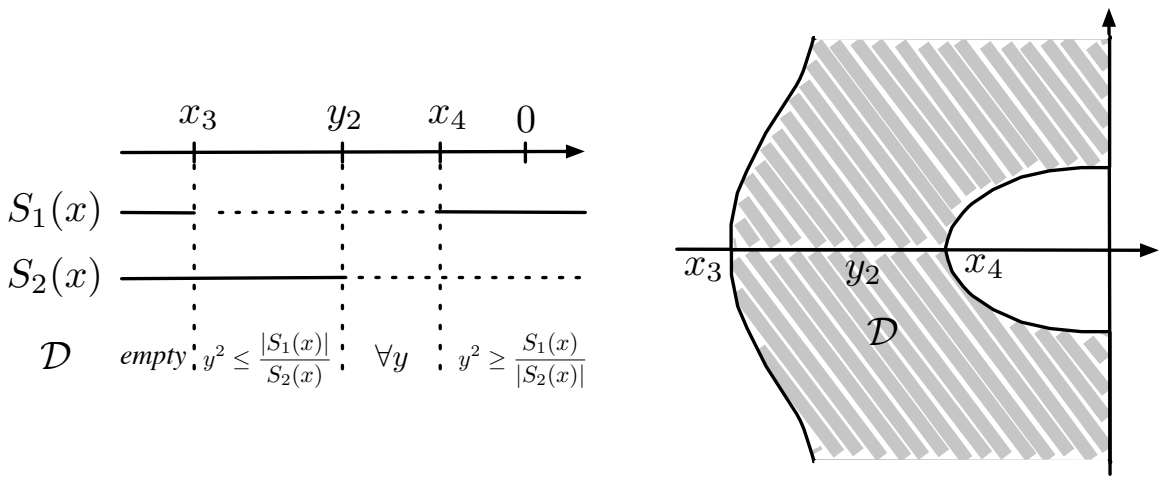

FIG. 6. The domain $\mathcal{D}:\left(D_{u} J_{22}+D_{v} J_{11}\right)^{2}-4 D_{u} D_{v} \operatorname{det} \mathbf{J}>0$ and $D_{u} J_{22}+D_{v} J_{11}>0, x_{3}<y_{2}<x_{4}$. Left panel: the signs of the functions $S_{1}(x)$ and $S_{2}(x)$ for $x \leq 0$. Right panel: the corresponding shape of the $\mathcal{D}$ domain is displayed. The domain intercepts the $x$-axis over an extended zone. Turing patterns can hence develop on symmetric supports.
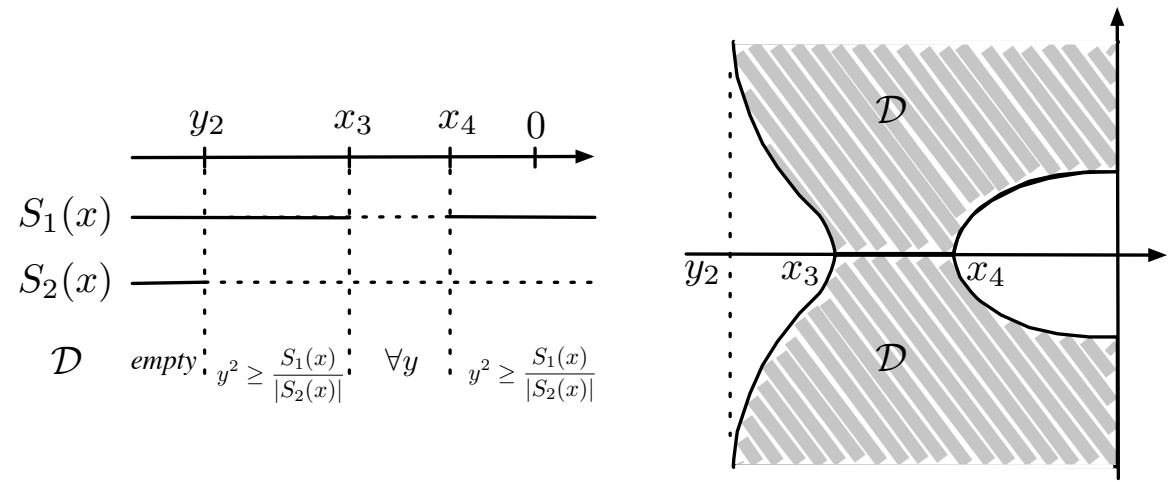

FIG. 7. The domain $\mathcal{D}:\left(D_{u} J_{22}+D_{v} J_{11}\right)^{2}-4 D_{u} D_{v} \operatorname{det} \mathbf{J}>0$ and $D_{u} J_{22}+D_{v} J_{11}>0, y_{2}<x_{3}<x_{4}$. Left panel: the signs of the functions $S_{1}(x)$ and $S_{2}(x)$ are shown for $x \leq 0$. Right panel: the corresponding profile of the $\mathcal{D}$ domain is depicted. Note the vertical asymptote and the fact that the domain has a limited extension on the horizontal axis. The domain intercepts the $x$-axis over an extended zone, and Turing patterns are consequently possible on symmetric supports.

where $\hat{S}_{2}(x)$ follows the definition of the coefficients $\hat{C}_{2 i}$. We can hence apply the above analysis to the scaled function $\hat{f}(x)$, which is regular in the limit $\epsilon \rightarrow 0$. In particular, we can now select the negative $\operatorname{root}(\mathrm{s})$ of Eq. (6) (where $S_{2}$ is replaced with $\hat{S}_{2}$ and where $\epsilon$ is set to zero), say $\hat{r}_{0}$. We can then compute $\hat{f}^{\prime}\left(\hat{r}_{0}\right)$ and conclude that $f^{\prime}\left(r_{0}\right)=\hat{f}^{\prime}\left(\hat{r}_{0}\right) / \epsilon$. That is, the optimal slope gets steeper as $\epsilon$ get closer to 0 . Moreover, for any given $\epsilon$ the slope is finite, as anticipated above.

\section{Allowing for diffusion and drift}

In the main text we have discussed the simplest scenario when species are only allowed to move clockwise. The aim of this section is to show that a similar analysis, based mainly on the spectral properties of the circulant matrices can be extended also to a more general case where species are allowed to move in both directions, across the ring, with assigned rates. In particular, we shall denote with $a$ and $b$ the rates respectively associ- ated to clockwise and anti-clockwise moves (see Fig. 10 a) for a schematic description). We can thus describe the process occurring at node $i$-th as follows:

$$
\begin{aligned}
\dot{u}_{i} & =D_{u} \sum_{j} \mathcal{L}_{i j} u_{j}=b u_{i-1}+a u_{i+1}-a u_{i}-b u_{i} \\
& =\frac{D_{u}(a+b)}{2}\left(u_{i-1}+u_{i+1}-2 u_{i}\right)+\frac{D_{u}(b-a)}{2}\left(u_{i-1}-u_{i}\right) \\
& =\sum_{j}\left(\frac{D_{u}(a+b)}{2} \mathcal{L}^{\mathcal{S}}{ }_{i j}+\frac{D_{u}(b-a)}{2} \mathcal{L}^{\mathcal{D}}{ }_{i j}\right) u_{j}
\end{aligned}
$$

where we have introduced the operators $\mathcal{L}^{\mathcal{S}}$ and $\mathcal{L}^{\mathcal{D}}$ to account for the symmetric and asymmetric components of the implemented transport mechanism where species are allowed to move in both directions, across the ring, with assigned rates, and we have considered also $a+b=1$. Let us notice that the Laplacian matrix is still circulant and observation which can be exploited to generalized to the current setting the analysis reperted in the main paper. To illustrate how the presence of diffusion shapes the conditions for the instability we refer to Figure 10 

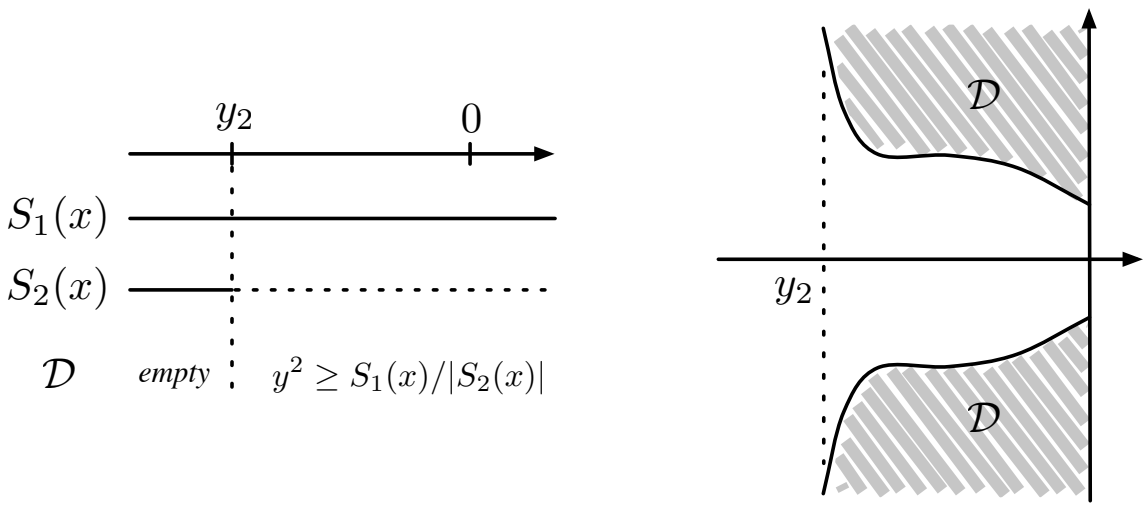

FIG. 8. The domain $\mathcal{D}:\left(D_{u} J_{22}+D_{v} J_{11}\right)^{2}-4 D_{u} D_{v} \operatorname{det} \mathbf{J}<0$ Left panel: the signs of the functions $S_{1}(x)$ and $S_{2}(x)$ are shown for $x \leq 0$. Right panel: the corresponding shape of the $\mathcal{D}$ domain is given. Note the vertical asymptote and the fact that the domain displays a limited horizontal extension. The domain does not intercept the $x$-axis. Turing patterns cannot set in when the system is made to evolve on a symmetric support.

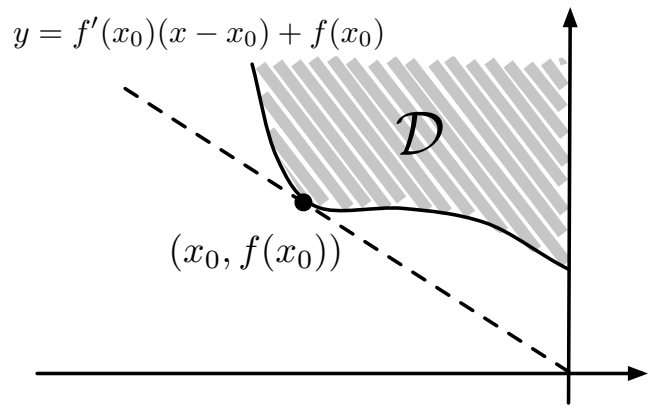

FIG. 9. Tangent to $\mathcal{D}$ : A schematic rapresentation of the tangent line passing through the origin.

b). As in the case discussed in the main text where only the drift term is considered, even in the case when the diffusion is present the eigenvalues of the Laplacian $\mathcal{L}$ fill an ellipse in the complex reference plane. This fact follows from the formula:

$$
\Lambda^{(\alpha)}=a+b+(a+b) \cos (2 \alpha / n)+i(b-a) \sin (2 \alpha / n),
$$

which returns the eigevalues of a circulant matrix. Here $n$ stands for the number of cells that compose the one dimensional lattice. Owing to the particular distribution of the eigenvalues, one readily extends the reasoning developed in the main paper to the general setting when symmetric and asymmetric transport are simulataneosly accounted for. In the pure drift case $(a=0, b \neq 0$ or $b=0, a \neq 0)$ the ellipse becomes a perfect circle. The asymmetry driven patterns fade aways only in the limiting setting when $a=b$, i.e. when the spectrum of the Laplacian $\mathcal{L}$ is real.
[1] S. Kondo and R. Asai, Nature 376, 765 (1995).

[2] J. D. Murray, Sci. Am. 258, 80 (1988).

[3] C. Klausmeier, Science 284, 1826 (1999).

[4] J. L. Maron and S. Harrison, Science 278, 1619 (1997).

[5] M. Rietkerk and J. van de Koppel, Trends Ecol. Evol. 23, 169 (2008).

[6] A. M. Turing, Phil. Trans. R. Soc. B 237, 37 (1952).

[7] P. Ball, The self-made tapestry: Pattern formation in Nature, 1st ed. (Oxford University Press, 1999).

[8] J. D. Murray, Mathematical biology II: Spatial models and biomedical applications (Springer-Verlag, 2001).

[9] G. Nicolis and I. Prigogine, Self-organization in nonequi- librium systems: From dissipative structures to order through fluctuations (J. Wiley \& Sons, 1977).

[10] The loss of stability of an homogeneous equilibrium, as triggered by an external perturbation, will be referred to as a Turing instability, as an natural extension of the original model discussed in [6].

[11] A. Gierer and H. Meinhardt, Kybernetik 12, 30 (1972).

[12] H. G. Othmer and L. E. Scriven, J. theor. Biol. 32, 507 (1971).

[13] H. Nakao and A. S. Mikhailov, Nat. Phys. 6, 544 (2010).

[14] R. S. Shaw, N. Packard, M. Schroter, and H. L. Swinney, PNAS 104, 9580 (2007). 

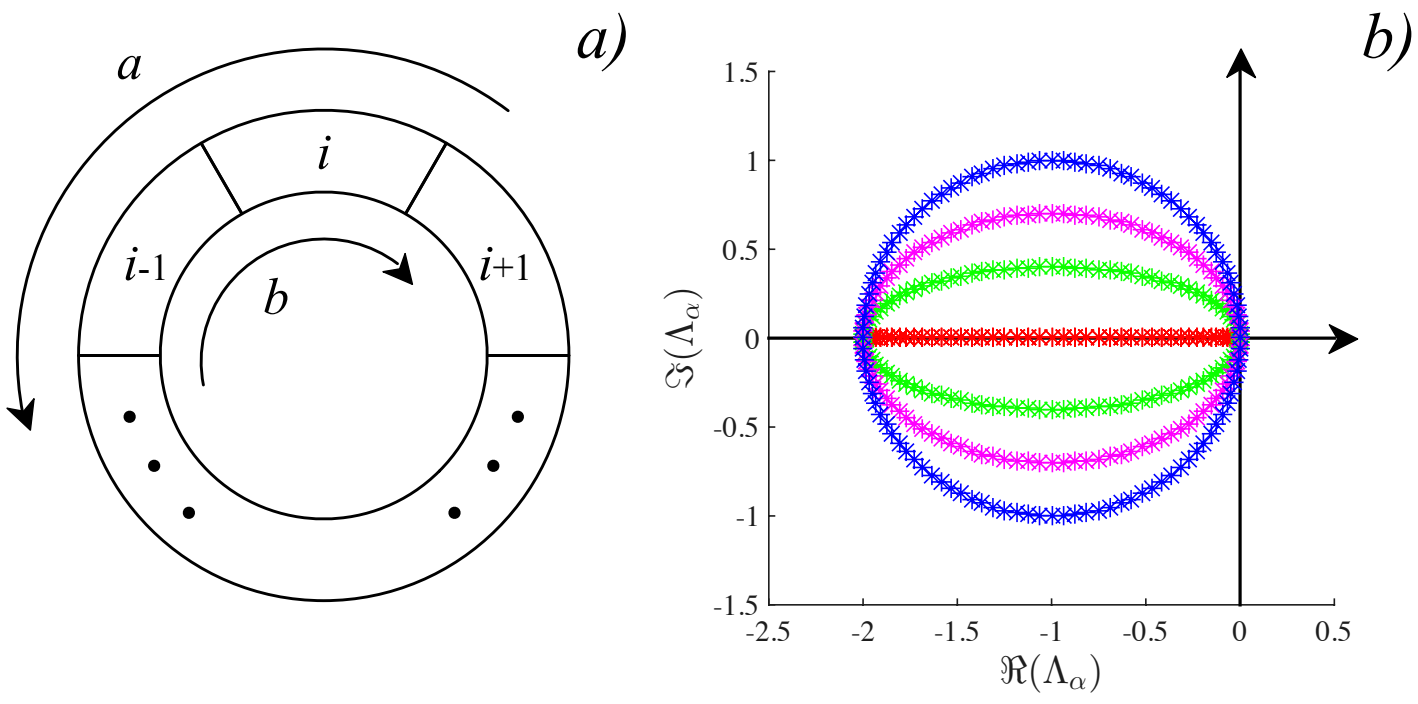

FIG. 10. The case of asymmetric transport where both diffusion and drift are present $a$ ) We show the transport formulation for the general case when the chemical move to both directions with different speeds $a$ and $b$. $b$ ) The Lapalcian spectrum for a ring of 100 cells for different choices of the parameters $(a=b=0.5$ red, $a=0.3, b=0.7$ green, $a=0.15$, $b=0.85$ magents and $a=0, b=1$ blue).

[15] K. S. Kim, I. S. Davis, P. A. Macpherson, T. J. Pedley, and A. E. Hill, Proc. R. Soc. A 461, 273 (2005).

[16] J. C. Dallon and H. G. Othmer, Phil. Trans. R. Soc. Lond. B 352, 391 (1997).

[17] M. Asllani, R. Lambiotte, and T. Carletti, Sci. Adv. 4, eaau9403 (2018).

[18] M. Postma, J. Roelofs, J. Goedhart, T. W. J. Gadella, A. J. W. G. Visser, and P. J. M. V. Haastert, Mol. Biol. Cell 14, 5019 (2003).

[19] A. B. Rovinsky and M. Menzinger, Phys. Rev. Lett. 96, 1193 (1992).

[20] A. B. Rovinsky and M. Menzinger, Phys. Rev. Lett. 70, 778 (1993).

[21] M. Asllani, J. D. Challenger, F. S. Pavone, L. Sacconi, and D. Fanelli, Nature Commun. 5, 4517 (2014).

[22] The eigenvalues comes in conjugate pairs. In what it follows we shall refer to the eigenvalue in the pair that displays positive imaginary part.

[23] R. Schnabel, M. Bischoff, A. Hintze, A.-K. Schulz, A. Hejnola, H. Meinhardt, and H. Hutter, Dev. Biol. 294, 418 (2006).
[24] M. Asllani, D. M. Busiello, T. Carletti, D. Fanelli, and G. Planchon, Sci. Rep. 5, 12927 (2015).

[25] P. Ciarletta, V. Balbi, and E. Kuhl, Phys. Rev. Lett. 113, 248101 (2014).

[26] J. D. Murray, J. Theor. Biol. 88, 161 (1981).

[27] For a general spatial arrangement, the eigenvalues of the Laplacian tend to populate a bounded domain of the semi-negative defined portion of the complex plane (a domain which can be ultimately delimited by the Gershgorin circles). The frontier of this compact domain plays the role of the unitary circle, where eigenvalues are positioned when dealing with a circulant matrix, hence periodic boundary condition. This effect is made visible in Fig. 1 of [21].

[28] J. M. Pringle, A. M. H. Blakeslee, J. E. Byers, and J. Roman, PNAS 108, 15288 (2011).

[29] O. Sporns, Networks of the Brain (MIT Press, 2010).

[30] In fact $-\operatorname{tr} \mathbf{J} /\left(D_{v}+D_{u}\right)$ is a double root of $S_{1}$, hence a simple root of $S_{1}^{\prime}$. Inserting $x_{0}=-\operatorname{tr} \mathbf{J} /\left(D_{v}+D_{u}\right)$ into Eq. (6) yields the identity $0=0$. 